Purdue University Purdue e-Pubs

7-24-2003

\title{
Downstream oxygen etching characteristics of polymers from the parylene family
}

Russell R. A. Callahan

Kristin G. Pruden

Gregory B. Raupp

Stephen P. Beaudoin

School of Chemical Engineering, Purdue University, sbeaudoi@purdue.edu

Follow this and additional works at: http://docs.lib.purdue.edu/nanodocs

Callahan, Russell R. A.; Pruden, Kristin G.; Raupp, Gregory B.; and Beaudoin, Stephen P., "Downstream oxygen etching characteristics of polymers from the parylene family" (2003). Other Nanotechnology Publications. Paper 10.

http://docs.lib.purdue.edu/nanodocs/10

This document has been made available through Purdue e-Pubs, a service of the Purdue University Libraries. Please contact epubs@purdue.edu for additional information. 


\title{
Downstream oxygen etching characteristics of polymers from the parylene family
}

\author{
Russell R. A. Callahan, Kristin G. Pruden, Gregory B. Raupp, and Stephen P. Beaudoin ${ }^{a)}$ \\ Department of Chemical and Materials Engineering, Arizona State University, Tempe, \\ Arizona 85287-6006
}

(Received 2 August 2002; accepted 19 May 2003; published 24 July 2003)

\begin{abstract}
As dictated by the International Technology Roadmap for Semiconductors, there is an immediate need to develop low dielectric materials for use in metalization and packaging schemes in integrated circuits. The etching characteristics of a family of low dielectric polymers, the parylenes, are discussed. These are good models for polymer dielectrics, and are attractive for packaging applications. Three types of parylene are studied: parylene-N, parylene-C, and parylene AF- 4 . Parylene films on silicon substrates were etched in a downstream microwave oxygen plasma system. The goal was to characterize the chemical reactions that occurred on the parylene in the afterglow of the microwave oxygen plasma. The effect of temperature on the etch rate of each polymer was studied and an apparent activation energy was determined. The apparent activation energy for the etch process is approximately $7.0 \mathrm{kcal} / \mathrm{mol}$ for each polymer. Infrared analysis showed carbonyl formation during etching in the parylene-N and $-\mathrm{C}$. Based on these analyses and the calculated activation energies, it was determined that a likely rate-limiting step in the etching was the ring opening. (C) 2003 American Vacuum Society. [DOI: 10.1116/1.1591744]
\end{abstract}

\section{INTRODUCTION}

Members of the parylene family of polymers are candidates for use as dielectrics or packaging materials in integrated circuits, ${ }^{1,2}$ as bio-compatible coatings for in vivo devices, and in microelectromechanical system (MEMS) and bio-MEMS applications. ${ }^{3}$

Parylenes are vapor deposited using a technique developed by Gorham. ${ }^{4}$ The deposition process is best described as vapor deposition polymerization (VDP). ${ }^{3}$ Figure 1 shows a schematic of the molecular reaction sequence for $\operatorname{poly}(p$ xylylene), parylene-N. A dimer molecule, di-p-xylylene, is evaporated at roughly 0.1 Torr and $120^{\circ} \mathrm{C}$. The evaporated dimer flows into a pyrolysis chamber kept at a temperature from 650 to $700{ }^{\circ} \mathrm{C}$. The dimer cracks inside the chamber to form $p$-xylylene monomer units. The monomer flows into the deposition chamber, condenses on any surface below a certain threshold temperature that depends on the type of parylene, and polymerizes.

Other variations of parylene exist and include parylene-C (poly(2-chloro-p-xylylene)) and parylene AF-4 (poly(tetrafluoro- $p$-xylylene)). The chemical structures of parylene $\mathrm{C}$ and parylene AF-4 are shown in Fig. 2. The monomer unit is basically the same for each parylene. Parylene-C adds a chlorine atom to the ring. Parylene AF-4 substitutes fluorine in the place of every hydrogen on the ethyl carbons which link the rings. Table I compares the physical properties of the three types of parylene. The water vapor transmission rate for parylene AF-4 is not reported, but the films exhibit very low moisture absorption $(<0.1 \%){ }^{5}$

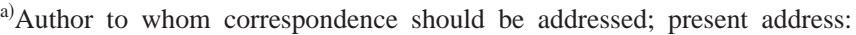
Forney Hall of Chemical Engineering, Purdue University, 480 Stadium Mall Drive, West Lafayette, IN 47907-2100; electronic mail: steve.beaudoi@purdue.edu
}

\section{EXPERIMENTAL METHODS}

The experimental apparatus used in this work is the same as reported elsewhere. ${ }^{6}$ Figure 3 shows the layout of the chamber and plasma system. The pressure in this system was controlled with an MKS (Type 253A) throttle valve along with an MKS (Type 127) capacitance manometer, and an MKS (PDR-C-2C) pressure readout and MKS (Type 252) controller. The etch chamber was made of stainless steel, and was 6 in. in diameter. Each parylene-N sample was $3.6 \mathrm{~cm}^{2}$. Samples were mounted on the face of a half cylinder of aluminum that was heated using two cartridge heaters. 250 $\mathrm{W}$ of applied power were used in all etching experiments.

The etching characteristics of parylene- $\mathrm{N}$, parylene- $\mathrm{C}$ and parylene AF-4 were studied. Blanket parylene films were deposited onto intrinsic double polished silicon (100) wafers by Para Tech, Inc using a VDP process.

The polymer film thickness and optical properties were characterized using a J.A. Woollam (M44) spectroscopic ellipsometer. The parylene films were modeled as anisotropic films using Cauchy expressions for the in-plane and out-ofplane constants. The Cauchy expression is

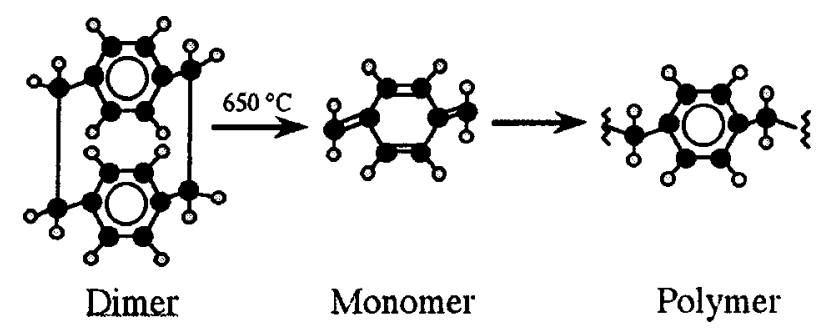

FIG. 1. Molecular representation of the parylene deposition process. 


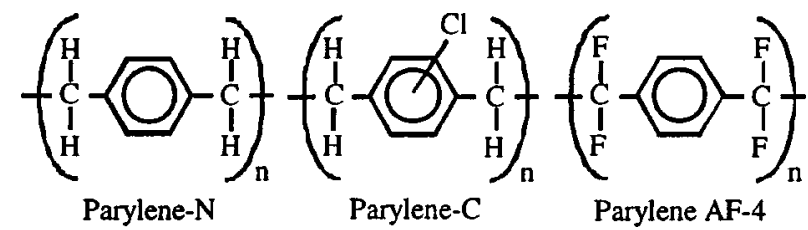

FIG. 2. Molecular structure of parylene-N, parylene-C, and parylene AF-4.

$$
n=A+\frac{B}{\lambda^{2}}+\frac{C}{\lambda^{4}},
$$

where $n$ is the index of refraction, $\lambda$ is the wavelength of light in microns, and $A, B$, and $C$ are the Cauchy coefficients. The $C$ coefficient was set to zero. The addition of the coefficient did not improve the agreement between the model and the experimental data. Table II shows the Cauchy coefficients determined using the ellipsometer data which includes del and psi measurements for 44 wavelengths of light and three angles of incidence. The Cauchy coefficients were used to calculate the index of refraction of the three films over the range of wavelengths used by the ellipsometer. Figure 4 shows the index of refraction for both the isotropic and uniaxial planes for the three films as a function of wavelength. Of the three films, the parylene AF-4 is the most anisotropic since the difference between the isotropic and uniaxial index of refraction is the greatest. The parylene-C film is the least anisotropic. While the values for the in-plane index of refraction for parylene- $\mathrm{N}$ and parylene- $\mathrm{C}$ are similar, the index of refraction for the parylene AF-4 is much lower.

The thickness of the films was measured before and after etching. The difference between the pre- and postetch thickness was divided by the etching time in order to determine the average etch rate.

\section{RESULTS}

\section{A. Etch rate evaluations}

The etch rates of the three polymers were studied as a function of temperature at a flow rate of $125 \mathrm{sccm}$ of oxygen and $250 \mathrm{~W}$ of applied plasma power. The data are shown in Fig. 5. The error bars represent the uncertainty in the thickness measurement. The etch rates for parylene-N and parylene-C are similar. At the low temperature, $373 \mathrm{~K}$, the etch rate is around $7 \mathrm{~nm} / \mathrm{min}$. An increase of $150 \mathrm{~K}$ results in an increase in the etch rate of nearly $100 \mathrm{~nm} / \mathrm{min}$. The etch rate of parylene AF-4 is lower than the etch rates of

TABLE I. Comparison of physical properties of three different types of parylene. $^{\mathrm{a}}$

\begin{tabular}{|c|c|c|c|}
\hline Property & Parylene-N & Parylene-C & $\mathrm{AF} 4$ \\
\hline Dielectric constant & 2.65 & 2.95 & 2.28 \\
\hline Water vapor transmission ${ }^{\mathrm{b}}$ & 1.5 & 0.14 & N/A \\
\hline Melting point $\left({ }^{\circ} \mathrm{C}\right)$ & 410 & 290 & 510 \\
\hline
\end{tabular}

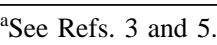

${ }^{\mathrm{b}} \mathrm{mol} / 100$ in. $^{2}$ in $24 \mathrm{~h}, 37^{\circ} \mathrm{C}, 90 \%$ relative humidity.

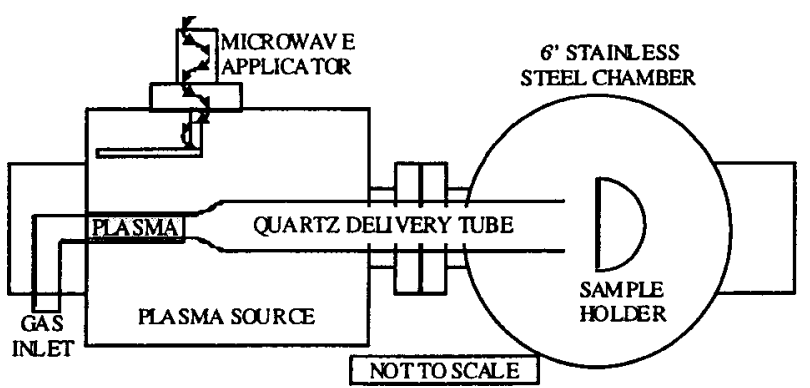

FIG. 3. Schematic of the downstream etching system.

parylene-N and parylene-C. The etch rate data was plotted on an Arrhenius style plot where the natural log of the etch rate was plotted as a function reciprocal temperature, as shown in Fig. 6. Included in the graph are the individual experimental data points and the best fit line for the experimental data. From the slope of the best fit lines, an apparent activation energy can be determined for each etch process. We call the activation energy "apparent" since a true activation energy is for a reaction rate constant, not for a rate. The best fit lines are nearly parallel suggesting that the apparent activation energy is similar for each polymer. The extracted activation energy for each film is shown in Fig. 7. The error bars represent the $95 \%$ confidence interval for the slope of the bestfit line used to determine the activation energy. The measured activation energies for all three polymers fall within the error reported for each activation energy measurement.

\section{B. Film analysis}

In order to further understand the reactions occurring during the etching process, infrared spectroscopy was performed using a Nicolet Nexus 870 spectrometer in transmission mode. First, a background spectrum of a bare double polished silicon sample was obtained. This background spectrum was subtracted from the spectra of the polymer films on top of double polished silicon substrates. The baseline was corrected to remove the sine wave created by the interference effect of the infrared light reflecting inside of the film. A comparison between the three unetched parylene polymers is shown in Fig. 8. The peak identification for all of the parylenes is in Table III.

The spectra of parylene- $\mathrm{N}$ and parylene- $\mathrm{C}$ are very similar. The $1513 \mathrm{~cm}^{-1}$ peak representing aryl carbon stretching

TABLE II. Comparison of the Cauchy coefficients used in the ellipsometry modeling.

\begin{tabular}{lcl}
\hline \hline & $A$ & $B$ \\
\hline Pa-N Isotropic & 1.623 & 0.0133 \\
Pa-N Uniaxial & 1.567 & 0.0114 \\
$\mathrm{~Pa}-\mathrm{C}$ Isotropic & 1.612 & 0.0129 \\
$\mathrm{~Pa}-\mathrm{C}$ Uniaxial & 1.599 & 0.0112 \\
$\mathrm{~Pa}-\mathrm{F}$ Isotropic & 1.522 & 0.0144 \\
$\mathrm{~Pa}-\mathrm{F}$ Uniaxial & 1.432 & 0.00487 \\
\hline \hline
\end{tabular}




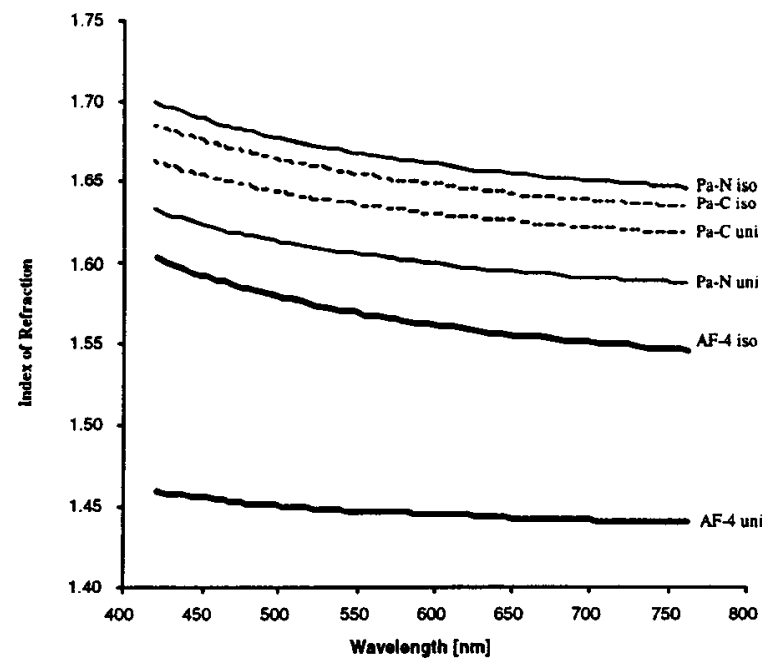

FIG. 4. Index of refraction for the three parylene films as a function of light wavelength.

in the parylene-N spectrum has shifted to $1495 \mathrm{~cm}^{-1}$ in the parylene-C spectrum. The ring hydrogen rocking peak is not present and the appearance of a peak at $1051 \mathrm{~cm}^{-1}$ is attributed to chlorine bonding on the ring. The $821 \mathrm{~cm}^{-1}$ peak is representative of two adjacent hydrogen atoms bonded to a ring. This peak shifts to $825 \mathrm{~cm}^{-1}$ in the parylene-C spectrum while a new peak at $877 \mathrm{~cm}^{-1}$ is representative of a single adjacent hydrogen on the ring, which in this case is adjacent to a chlorine and ethyl group. While the parylene-N and parylene-C spectra show the same peaks in the 2800$3100 \mathrm{~cm}^{-1}$ range, the relative ratio of the peaks changes. The number of aryl carbon-hydrogen bonds is reduced in parylene-C compared to parylene- $\mathrm{N}$ since the chlorine replaces one hydrogen on the ring. The peak at $3017 \mathrm{~cm}^{-1}$ is representative of aryl carbon-hydrogen bonding while the other two peaks are from the carbon-hydrogen bonds on the ethyl carbons. When the two spectra are compared, the ratio of the area of the aryl bonding peak, $3017 \mathrm{~cm}^{-1}$, to the $\mathrm{C}-\mathrm{H}$

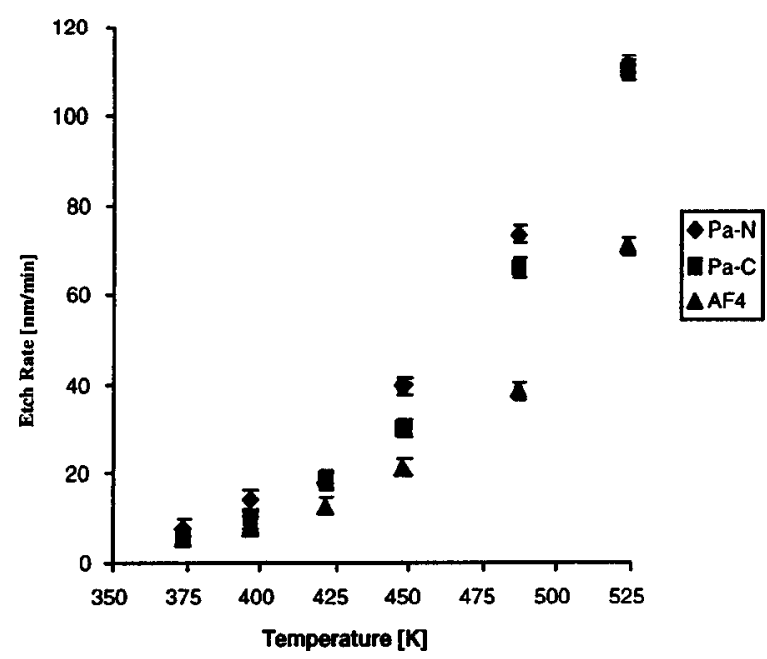

FIG. 5. Etch rate of three different parylenes as a function of temperature.

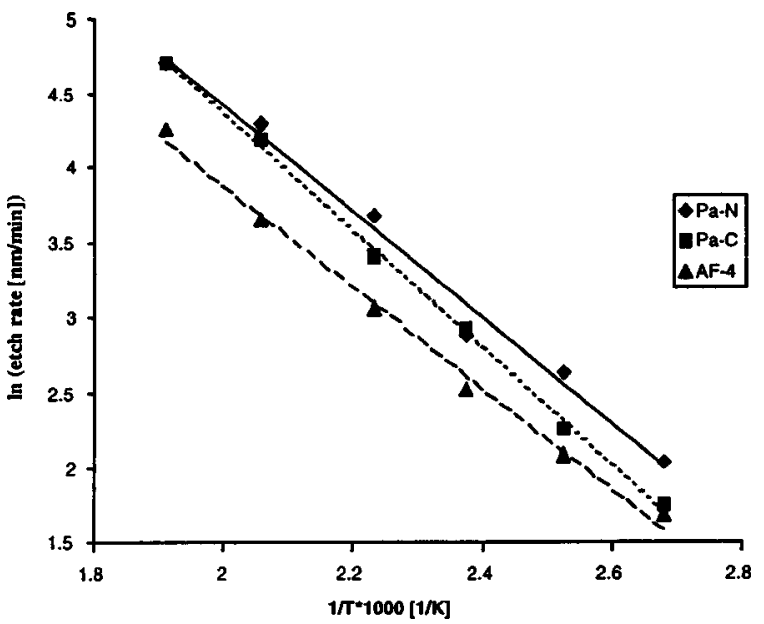

FIG. 6. Arrhenius style plot of the etch rate vs temperature data for three different parylene polymer films.

bonding on the ethyl carbons bonding, 2923 and $2860 \mathrm{~cm}^{-1}$, is reduced in the parylene-C spectrum when compared to the parylene-N spectrum.

Compared to the parylene- $\mathrm{N}$ and $-\mathrm{C}$ spectra, the spectrum for parylene AF-4 shows that the peaks in the range of 2860$3020 \mathrm{~cm}^{-1}$ and at $1452 \mathrm{~cm}^{-1}$ have nearly disappeared since fluorine has replaced hydrogen on the ethyl carbons. Minor absorption can be seen, but most likely this is slight parylene-N contamination since the parylene AF-4 was deposited in the same reactor which was used for parylene-N deposition. The aryl carbon stretching peak has shifted to $1491 \mathrm{~cm}^{-1}$. Three new peaks appear in the range of 1170 $1306 \mathrm{~cm}^{-1}$. These peaks have not been identified, but are thought to be either carbon-fluorine bonding or ring hydrogen rocking. The peak at $944 \mathrm{~cm}^{-1}$ is thought to be carbonhydrogen bending from the benzene ring. Normally, this peak would be at $821 \mathrm{~cm}^{-1}$ since the benzene is para substituted. It is assumed that the fluorine is causing the shift.

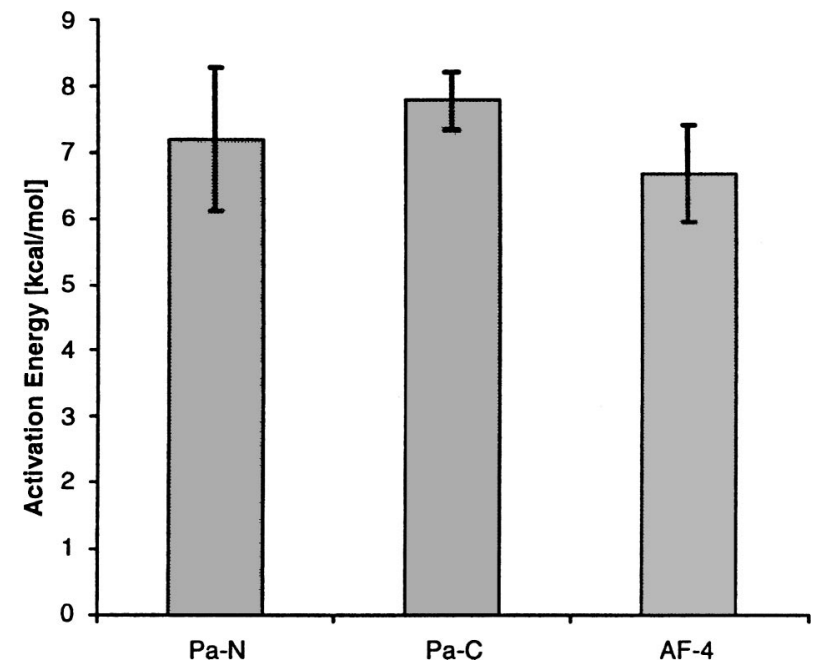

FIG. 7. Apparent activation energy for the downstream etching of three different parylene films. 


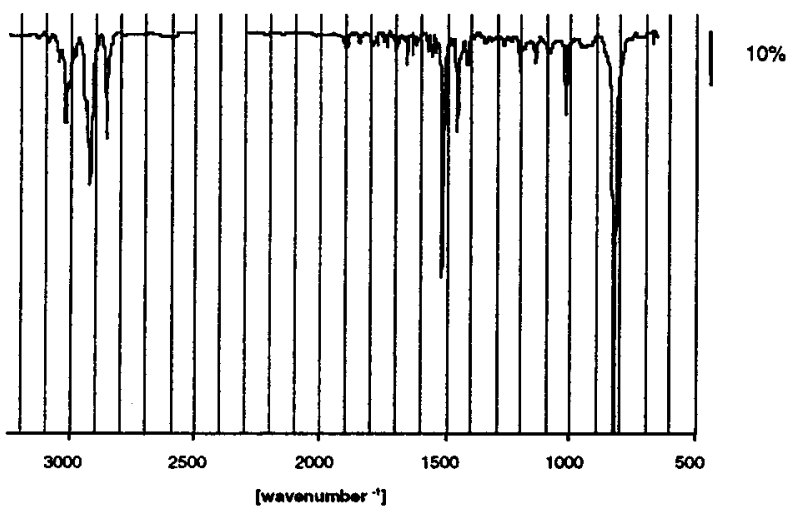

(a)

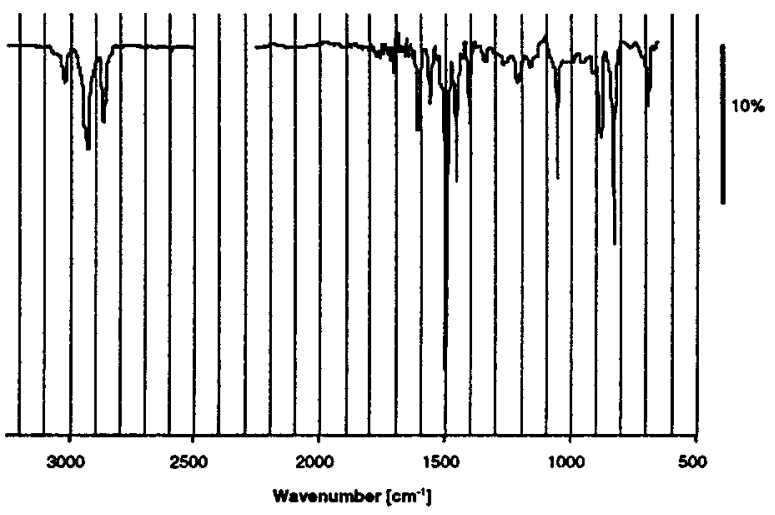

(b)

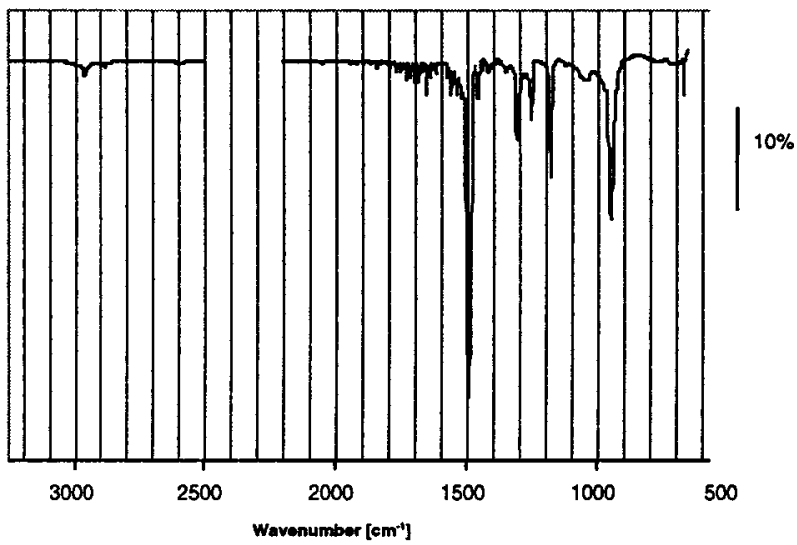

(c)

FIG. 8. Comparison of infrared spectra for: (a) unetched parylene-N, (b) unetched parylene-C, and (c) unetched AF-4.

Figure 9 shows the infrared spectra for etched parylene-N, parylene-C, and parylene AF-4 films over the range of 1600$1800 \mathrm{~cm}^{-1}$. If any new carbon-oxygen bonding was created during the etching process, the new peaks would appear in this range. To eliminate misinterpretation of infrared (IR) peaks that may be caused by water vapor, a water vapor transmission spectrum was subtracted from each of the etched parylene spectra. The etched spectra are for roughly $100 \mathrm{~nm}$ thick films. The etched parylene- $\mathrm{N}$ and parylene-C spectra show new peaks around $1700 \mathrm{~cm}^{-1}$. Absorbance peaks at wave numbers between 1600 and $1750 \mathrm{~cm}^{-1}$ are
TABLE III. Peak assignment for principal infrared absorption bands of parylene-N, parylene-C, and AF-4.

\begin{tabular}{lcc}
\hline \hline Polymer & Wave number $\left(\mathrm{cm}^{-1}\right)$ & Group \\
\hline $\mathrm{Pa}-\mathrm{N}, \mathrm{Pa}-\mathrm{C}, \mathrm{AF}-4$ & $1491-1514$ & Aryl C-C stretching \\
$\mathrm{Pa}-\mathrm{N}, \mathrm{Pa}-\mathrm{C}$ & 1450 & $\mathrm{C}-\mathrm{H}_{2}$ bending \\
$\mathrm{Pa}-\mathrm{N}, \mathrm{Pa}-\mathrm{C}$ & 2860 & $\mathrm{C}-\mathrm{H}$ stretching \\
$\mathrm{Pa}-\mathrm{N}, \mathrm{Pa}-\mathrm{C}$ & 2923 & $\mathrm{C}-\mathrm{H}$ stretching \\
$\mathrm{Pa}-\mathrm{N}, \mathrm{Pa}-\mathrm{C}$ & 3017 & Aryl C-H stretching \\
$\mathrm{Pa}-\mathrm{N}, \mathrm{Pa}-\mathrm{C}$ & $821-825$ & Two adjacent C-H bending on \\
& & benzene ring \\
$\mathrm{Pa}-\mathrm{N}$ & 1020 & Aryl H rocking \\
$\mathrm{Pa}-\mathrm{C}$ & 877 & One adjacent C-H bending on \\
& & benzene ring \\
$\mathrm{Pa}-\mathrm{C}$ & 1050 & Aryl Cl \\
$\mathrm{AF}-4$ & 944 & Substituted benzene C-H \\
& & bending \\
\hline \hline
\end{tabular}

attributed to carbonyl groups, with carboxylic groups assigned to peaks between 1690 and $1750 \mathrm{~cm}^{-1}$ and aldehydic groups assigned to peaks between 1650 and $1690 \mathrm{~cm}^{-1}$. Peaks in both the carboxylic and aldehydic range are observed. The observed carbonyl absorptions are small compared to the normal parylene absorptions (e.g., $\mathrm{C}-\mathrm{C}$ bonding in the parylene rings), indicating that only a small amount of carbonyl species reside in the etched parylene. The etched parylene AF-4 does not show any peaks in the carbonyl region. It is hypothesized that the detachment of oxygenated species from the parylene AF-4 may have been very fast compared to the rate of opening of the rings, so that these species are not detected in the residual film. As mentioned above, transmission IR spectroscopy was performed to detect oxygenated species on the films, and this approach may not detect small levels of oxygen incorporation into the surface region of the film. In the case of the AF-4, oxygen may be incorporated into the films, but not to the extent required for detection by the IR.

\section{Etch mechanisms}

The etch rate data and IR analysis provide insight into the etch process. In a previous article, ${ }^{6}$ the preliminary steps of the etching process of parylene-N were discussed. There, the etching process was hypothesized to start with hydrogen ab-

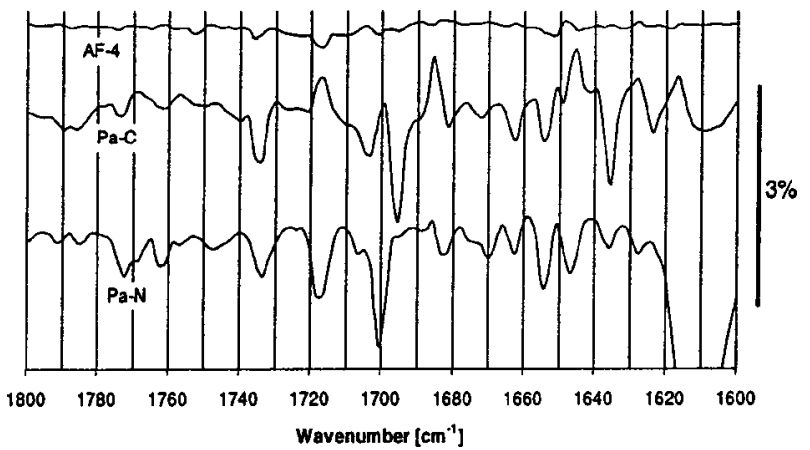

FIG. 9. Transmission infrared spectra of etched parylenes with water transmission peaks subtracted. 
straction from the ethyl carbons between the rings in the parylene. Next, an oxygen molecule or atom adsorbed on the reactive site. If an oxygen molecule adsorbed, a peroxy radical was formed. This species could rearrange to form a carboxylic group which could volatilize to form carbon dioxide. If an oxygen atom adsorbed, the resulting carbonyl species could rearrange to form an aldehydic group which could volatilize to form carbon monoxide. After the rearrangement and volatilization of the carbon monoxide or carbon dioxide, a radical site is formed on the aromatic ring. If another hydrogen is abstracted adjacent to the radical site and an oxygen molecule or atom adsorbs, the ring opens and carboxylic or aldehydic groups are left on the resultant chain. The infrared analysis here shows carboxylic group formation, which supports this process.

The mechanisms for parylene-C etching are believed to be similar to those for parylene-N. Just like parylene-N, a hydrogen can easily be abstracted from the ethyl carbons which leads to the reactive site formation on the ring and eventually ring opening, after a second hydrogen is abstracted from the ring. Again, the infrared analysis showed evidence of formation of carboxylic groups on the polymer. The etching of parylene- $\mathrm{C}$ differs from that of parylene- $\mathrm{N}$ due to the presence of the chlorine on the ring. An oxygen radical cannot abstract a chlorine atom from the ring. Therefore, that carbon site does not become a reactive site that could lead to ring opening. Consequently, five of the six carbons on the ring are available as reactive sites and the etch rate should be $5 / 6$ $(0.83)$ of the parylene- $\mathrm{N}$ etch rate if the ring opening process is the rate limiting step. For the six etch rates measured in Fig. 5, the parylene-C etch rate is 0.86 times the parylene-N etch rate with a standard deviation of 0.15 . The etching process for parylene AF-4 is thought to be slightly different from that of the parylene-N, as the $\mathrm{F}$ atoms on the ethyl carbons between the ring species in the parylene AF-4 will not be abstracted by oxygen atoms. Since parylene AF-4 does not have hydrogen on the ethyl carbons, and since the fluorine is not abstracted by oxygen atoms, reactive sites on the aromatic ring cannot be formed through the loss of ethyl carbons. Instead, two adjacent hydrogen atoms have to be abstracted from the ring. Since only four of the six sites on the ring are now available to participate in the ring opening process, the etch rate should be 4/6 (0.66) of the parylene-N etch rate. Experimentally, the etch rate is 0.61 times the etch rate of parylene-N with a standard deviation of 0.08 . A summary of the results is found in Fig. 10. The relationship between carbon substitution and etch rate supports the proposed ring opening etch mechanism.

In a previous article, ${ }^{6}$ the comparison between the experimental and model data predicted a half order reaction rate dependence on the oxygen atom concentration. A fractional order can be explained by multiple reactions occurring with reactive intermediates. It is even possible that adsorption and desorption steps could be rate limiting. A series of steps which might lead to a fractional order dependence is the ring opening process suggested here.

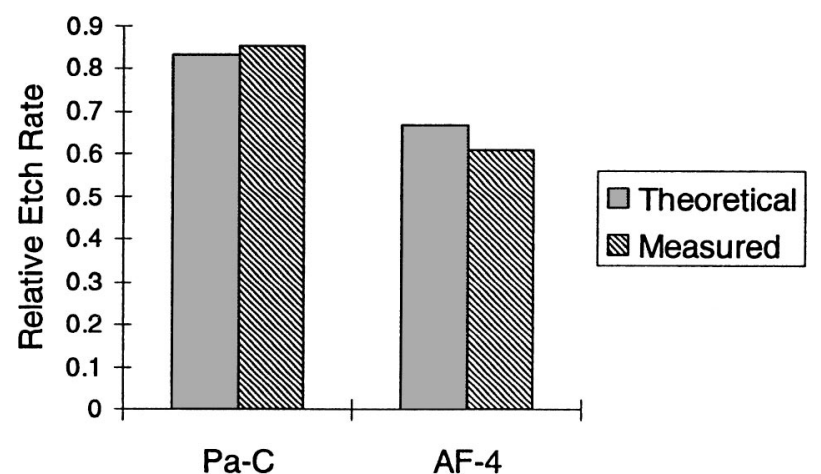

FIG. 10. Comparison of the theoretical and measured etch rates of parylene-C and parylene $\mathrm{AF}-4$ relative to parylene-N.

\section{CONCLUSIONS}

The etching characteristics of three polymers from the parylene family have been investigated in the afterglow from a microwave oxygen plasma. First, the etch rate of the three polymers as a function of temperature was investigated. The relative etch rate of parylene-C to parylene- $\mathrm{N}$ was on average 0.83 over a $150{ }^{\circ} \mathrm{C}$ temperature range, while the relative rate of parylene AF-4 to parylene-N was on average 0.61 over the same temperature range. Arrhenius-style plots were prepared to extract the apparent activation energies of the etching process for each polymer. The activation energies for each polymer fell within the experimental error of the others.

Since the polymers have the same activation energy and etch rates that vary directly with the number of abstractable hydrogen atoms that can lead to radicals on the conjugated rings, the activation energy is apparently for the ring opening process. As potential reactive sites are blocked by $\mathrm{Cl}$ or $\mathrm{F}$ species, the etch rate decreases proportionally.

\section{ACKNOWLEDGMENTS}

The authors would like to thank Ron Synowicki and Corey Bungay with J.A. Woollam for help with the ellipsometry modeling and Timothy Karcher with the Center for Solid State Science at Arizona State University for help with XPS. They are also grateful to Paratech, Inc. for depositing parylene onto silicon wafers. This work was funded by NSF/ EPA Grant No. CTS-9613377 and the NSF/SRC Center for Environmentally-Benign Semiconductor Manufacturing.

${ }^{1}$ J. Gaynor and A. Ralston, Semicond. Int. December, 73 (1997).

${ }^{2}$ L. Peters, Semicond. Int. September, 64 (1998).

${ }^{3}$ W. F. Beach, C. Lee, D. R. Bassett, T. M. Austin, and R. Olson, Encyclopedia of Polymer Science and Engineering, edited by J. I. Kroschwitz (Wiley, New York, 1985), p. 990.

${ }^{4}$ W. F. Gorham, U.S. Patent No. 3,342,754 (19 September, 1967).

${ }^{5}$ R. Sutcliffe, W. W. Lee, J. F. Gaynor, J. D. Luttmer, D. Martini, J. Kelber, and M. A. Plano, Appl. Surf. Sci. 126, 43 (1998).

${ }^{6}$ R. R. A. Callahan, G. B. Raupp, and S. P. Beaudoin, J. Vac. Sci. Technol. B 19, 725 (2001) 